\title{
Construction of conductive hydroxyethyl cellulose/soy protein isolate/polypyrrole composite sponges and their performances
}

\section{Yanteng Zhao}

Zhengzhou University First Affiliated Hospital

\section{Qiankun Yang}

Zhengzhou University First Affiliated Hospital

\section{Qianqian Cheng}

Wuhan University

Junjie Ai

Wuhan University

\section{Mengna Feng}

Yancheng Institute of Technology

\section{Chengshuang Wang}

Yancheng Institute of Technology

\section{Xianping Lv}

Zhengzhou University First Affiliated Hospital

\section{Meng He}

Yancheng Institute of Technology

Yun Chen ( $\nabla$ yunchen@whu.edu.cn )

Wuhan University https://orcid.org/0000-0002-5984-7455

\section{Research Article}

Keywords: hydroxyethyl cellulose, soy protein isolate, sponges, polypyrrole, electro-conductive, biocompatibility

Posted Date: May 3rd, 2021

DOI: https://doi.org/10.21203/rs.3.rs-412080/v1

License: (c) (1) This work is licensed under a Creative Commons Attribution 4.0 International License.

Read Full License 
Version of Record: A version of this preprint was published at Cellulose on July 14th, 2021. See the published version at https://doi.org/10.1007/s10570-021-04074-4. 


\section{Abstract}

In the present study, we have in situ synthesized polypyrrole (PPy) on the hydroxyethyl cellulose/soy protein isolate (HEC/SPI) sponges to construct electro-conductive HEC/SPI/PPy composite sponges (EHSS-Pn, $\mathrm{n} \otimes 0$ ). The composite sponges were characterized by Fourier transform infrared spectroscopy (FTIR), X-ray diffraction (XRD), scanning electron microscopy (SEM), conductivity and mechanical tests. The results indicated that EHSS-Pn still exhibit homogenous inter-connected macroporous structure for cell adhesion, proliferation and metabolism, indicating that the incorporation of PPy didn't break the original HEC/SPI sponge structure. The electrical conductivity and mechanical properties of the HEC/SPI sponge were improved significantly by the incorporation of PPy. Cytocompatibility and hemocompatibility of all the sponges were evaluated by a series of in vitro experiments. The results of MTT assay and cell direct contact tests showed that the introduction of PPy didn't cause any cytotoxicity and EHSS-Pn had good biocompatibility. Moreover, EHSS-Pn had good hemocompatibility and no significant side effects on the anticoagulant whole blood with the introduction of PPy. Therefore, the electro-conductive EHSS-Pn showed potential application in the tissue engineering field that requires electrical conductivity for stimulation or sensing such as neural tissue restoration.

\section{Introduction}

Electric signals could regulate our body functions such as neural communication and heartbeat through the transmitting of information from neuron to neuron by synapses or the production of electrical impulses that travel through the entire organ, respectively (Funk et al. 2009; Alegret et al. 2019). Moreover, some tissues such as bone marrow, could use conductivity to regenerate new tissue (Clayton et al. 2011). Therefore, electro-conductive biomaterials with proper conductivity have attracted great deal of interest in the tissue engineering filed because they could interact with electrically active biological tissues due to their inherent electrical conductivity in some biological functions, including cell adhesion, differentiation and migration, DNA synthesis, and protein secretion etc (Massoumi et al. 2020; Dong et al. 2019; Walker et al. 2019). Amongst, electro-conductive scaffolds (ECS) with 3D structures showed extraordinary advantages because they could not only provide 3D space for cell adhesion, proliferation and metabolism, but also exhibit electrical conductivity for stimulation or sensing (Alegret et al. 2019).

Recently, natural biopolymers based scaffolds, especially polysaccharide/protein systems, have attracted great interest due to their desirable characteristics such as cytocompatibility, hemocompatibility, histocompatibility and in vivo biodegradability and non-toxicity (Zhao et al. 2019; Baniasadi et al. 2015). However, it is nearly impossible to fabricate ECS by using natural polymers solely due to their inherent no or poor electrical conductivity, some electro-conductive materials such as carbon-based materials (e.g., carbon nanotube, carbon black and graphene) or electro-conductive polymers have to be incorporated to improve or render their conductivity (Alegret et al. 2019; Ahadian et al. 2017; Deng et al. 2019; Du e al. 2018; Bahrami et al. 2019). Electro-conductive polymers are an optimal choice for ECS due to their proper conductivity and high biocompatibility, and the potential candidates could be polyaniline (PANI) or aniline tetramer (Khorshidi and Karkhaneh 2018; Qu et al. 2019), PEDOT (Wang et al. 2017; Zamora-Sequeira et 
al. 2018; Kayser et al. 2018), polythiophene (PTh) (Abbasian et al. 2019) and polypyrrole (PPy) (Hung et al. 2018; Kashi et al. 2018) etc. Among the above electro-conductive polymers, PPy is one of the foremost promising polymers due to its reasonable conductivity, redox properties, environmental stability and biocompatibility, which could be incorporated into other polymers' matrix facilely through in situ polymerization (Sadeghi et al. 2018; Kim et al. 2018; Su et al. 2019; Min et al. 2018).

In our previous work, highly biocompatible hydroxyethyl cellulose (HEC)/soy protein isolate (SPI) composite sponges with uniformly inter-connected porous structure and a fluid responsive shape memory property were successfully fabricated, which could be used as blood-contacted scaffolds for subcutaneous defect filling and repair (Zhao et al. 2019; Zhao et al. 2018). As we mentioned above, the poor electrical conductivity had restricted their further applications in the biomedical fields such as muscle or neural repair. Thus, a worthwhile endeavor would be to construct a novel HEC/SPI based ECS to broaden their applications. In the present work, pyrrole was in situ polymerized in the HEC/SPI sponges, the effects of the incorporation of the resultant PPy on the structure and properties of the $\mathrm{HEC} / \mathrm{SPI}$ sponges were investigated systematically. The results showed that the resultant HEC/SPI/PPy sponges exhibited macro-porous structure, proper electrical conductivity and mechanical property, good cytocompatibility and hemocompatiblity, showing potential applications as 3D implants with electrical conductivity requirement (Milakin et al. 2020).

\section{Experimental Procedure}

\subsection{Materials}

HEC with the viscosity of $30000 \mathrm{mPa} \cdot \mathrm{s}$ was purchased from Shandong Head Reagent Co. Ltd. (Shandong, China). Commercial SPI $\left(\mathrm{M}_{\mathrm{w}}: 2.05 \times 10^{5} \mathrm{~g} / \mathrm{mol}\right)$ was obtained from DuPont-Zhengzhou Protein (Zhengzhou, China). Both HEC and SPI powders were dried in a vacuum dryer before use. Epichlorohydrin $(\mathrm{ECH}), \mathrm{NaOH}, \mathrm{FeCl}_{3}, \mathrm{CaCl}_{2}$, dimethyl sulfoxide (DMSO) and acetic acid were purchased from Sinopharm Chemical Reagent Co. Ltd. (Shanghai, China). Pyrrole and sodium dodecyl benzene sulfonate (SDBS) were bought from Sigma (USA). Modified Eagle's Medium (MEM), fetal bovine serum (FBS) and 3-[4,5dimethyl-2- thiazoly1]-2,5-diphenyl-2H-tetrazolium bromide (MTT) were obtained from Invitrogen Corporation (Gibco BRL, Grand Island, NY, USA). 3,3'-dioctadecyloxacarbocyanineperchlorate (DiO) for cell membrane staining were purchased from Beyotime Institute of Biotechnology (Jiangsu, China). Other chemicals were of analytical grade agents and used without further treatment.

\subsection{Preparation of the electro-conductive HEC/SPI/PPy sponges}

Firstly, pure HEC/SPI sponges were prepared according to our previous work (Zhao et al. 2019). Briefly, 5 $\mathrm{g}$ SPI powder were dispersed into $30 \mathrm{~g}$ deionized water to completely swollen with the following addition of $15 \mathrm{~g} \mathrm{NaOH}$ aqueous solution (5 wt\%) to obtain a viscous aqueous solution with $10 \%$ SPI content. $1 \mathrm{~g}$ HEC was dissolved in $49 \mathrm{~g}$ deionized water to obtain $2 \% \mathrm{HEC}$ aqueous solution. HEC and SPI solutions were mixed (with the dry weight ratio of 3 to 7 ) with the following addition of $\mathrm{ECH}$ ( $20 \%$ of the total weight of original SPI and HEC powders). Then, the degassed transparent HEC/SPI blend solutions were 
poured into square molds and kept in a refrigerator to complete the crosslinking process. The resultant $\mathrm{HEC} / \mathrm{SPI}$ composite hydrogels were frozen at $-20^{\circ} \mathrm{C}$ for $12 \mathrm{~h}$ and $-80^{\circ} \mathrm{C}$ for $24 \mathrm{~h}$ and then freeze-dried. The freeze-dried HEC/SPI samples were treated in a $5 \%$ acetic acid solution, rinsed completely and freeze-dried again to obtain the HEC/SPI composite sponges.

Pyrrole monomer was dissolved in ethanol to get a solution with the concentration of $100 \mathrm{~g} / \mathrm{L}$, and $\mathrm{FeCl}_{3}$ was also dissolved in ethanol to reach the final concentration of $1 \mathrm{~mol} / \mathrm{L}$. The above HEC/SPI composite sponges were pretreated by soaking in the above pyrrole solution firstly and then in the above $\mathrm{FeCl}_{3}$ solution for the same time ( $n$ ), this process was coded as one cycle. After one cycle, the pretreated $\mathrm{HEC} / \mathrm{SPI}$ composite sponges was treated in a $0.3 \mathrm{~mol} / \mathrm{L}$ SDBS solution for $12 \mathrm{~h}$. The resultant sponges were denoted as EHSS-Pn according to the above soaking time (n) in the pyrrole solution and $\mathrm{FeCl}_{3}$ solution, for example, EHSS-P6 corresponded to EHSS after being soaked in the pyrrole solution and $\mathrm{FeCl}_{3}$ solution for $6 \mathrm{~h}$ respectively. However, EHSS-P24 was obtained by two cycles of the soaking EHSS in the pyrrole solution and $\mathrm{FeCl}_{3}$ solution for $12 \mathrm{~h}$, respectively. Obviously, the $\mathrm{HEC/SPI}$ composite sponges without PPy could be denoted as EHSS-PO.

\subsection{Characterization}

FTIR spectra were measured with a FTIR spectrometer (TNZ1-5700) over the wave number range from 4000 to $400 \mathrm{~cm}^{-1}$. The EHSS-Pn sponges were cut into powder and vacuum-dried at $60{ }^{\circ} \mathrm{C}$ for $48 \mathrm{~h}$. The powders were respectively mixed with $\mathrm{KBr}$ to laminate for the measurements of FTIR. X-ray diffraction measurements were measured with a WAXD diffractometer (D8-Advance, Bruker,USA). The patterns with Cu K $\mathrm{a}_{\mathrm{a}}$ radiation $(\lambda=0.15406 \mathrm{~nm})$ at $40 \mathrm{kV}$ and $30 \mathrm{~mA}$ were recorded over the region of $2 \theta$ from 5 to $40^{\circ}$, scanning rate was $2 \%$ min. The dried EHSS-Pn sponges were cut into powders, and then the vacuum-dried powders were used for XRD analysis. The morphology of the EHSS-Pn sponges was observed on a scanning electron microscope (SEM, VEGA3, TESCAN, Czech Republic) with $20 \mathrm{kV}$ as the accelerating voltage. The surfaces of the sponges were coated with gold for SEM observation. EHSS-Pn were embedded with resin (hydroxyethyl methacrylate and butyl methacrylate) and sliced for ultrathin section, which were supported on carbon coated copper grids for TEM observation (JEOLJEM 2010 FEF).

The EHSS-Pn sponges were cut into disks with the diameter of $10 \mathrm{~mm}$, vacuumed dried at $60{ }^{\circ} \mathrm{C}$ for $4 \mathrm{~h}$ and then kept in a sealed container with $\mathrm{CaCl}_{2}$ for $5 \mathrm{~d}$, and the compressive strength of the resultant sponges were tested on a universal testing machine (CMT6503, Shenzhen SANS Test Machine, China) according to ISO527 - 1995 (E) at a speed of $1 \mathrm{~mm} / \mathrm{min}$. The mean values of the compressive strength of the sponges were calculated from three specimens. The conductivity of the above EHSS-Pn sponges disks (10 mm diameter) was tested by Four-Point Probes.

\subsection{Cytocompatibility Evaluation of EHSS-Pn}

The cytocompatibility is very important for the application of EHSS-Pn as biomaterials after the incorporation of PPy, which was evaluated by the MTT and direct cell culture methods according to ISO 10993-5: 2007, respectively (Zhao et al. 2018). 
EHSS-Pn were cut into $1 \mathrm{~cm} \times 1 \mathrm{~cm}$ blocks with the thickness of $0.2 \mathrm{~cm}$, which was packed with tin foil and treated at $121^{\circ} \mathrm{C}$ for totally sterilization before use. The sterilized EHSS-Pn sponges were cut into powder and then immersed in a MEM 1640 cell culture medium $(0.2 \mathrm{~g}$ sponge $/ \mathrm{mL})$. After centrifugation at $1000 \mathrm{rpm}$ for $10 \mathrm{~min}$, the resultant supernatants of the above solutions were the corresponding extracts.

\section{Cell viability evaluation by MTT assay and direct contact method}

The experimental processes for cell viability evaluation by MTT assay and direct contact method were similar to our previous work (Zhao et al. 2018). Briefly, the suspension with the cell concentration of $5 \times 10^{3}$ cells $/ \mathrm{mL}$ was prepared and inoculated into 96 well culture plates. The complete medium with cells and single complete medium were coded as the negative control and the blank control (for calibration), respectively. After incubation for $24 \mathrm{~h}$, the original culture solution was discarded and PBS was added for rising. PBS culture solution and EHSS-Pn extract solution was added, the negative control was added with the same amount of medium. After incubating at $37^{\circ} \mathrm{C}$ for 24,48 and $72 \mathrm{~h}$, the cells were treated with $20 \mu \mathrm{L} /$ well of MTT $(5 \mathrm{mg} / \mathrm{mL})$ and incubated for another $4 \mathrm{~h}$. Then, the culture solution was removed carefully, and DMSO was added and vibrated for $10 \mathrm{~min}$. Absorbance values were read in triplicate at a test wavelength of $570 \mathrm{~nm}$ using a multiwell microplate reader (Tecan GENios, Tecan Austria GmbH, Salzburg, Austria). Cell viability was calculated using Eq. (1) (He et al. 2014):

Cell viability $(\%)=\left(A_{\text {test }} / A_{\text {control }}\right) \times 100(1)$

where $A_{\text {test }}$ and $A_{\text {control }}$ are the absorption values of the test and negative control groups, respectively.

The steam-sterilized EHSS-Pn sponges were put in a 24-well cell culture plate, respectively. $100 \mu \mathrm{L}$ cell suspension was dripped on the surface of EHSS-Pn, the cell suspension with the same volume was added in the blank well as the negative control. After incubation for $2 \mathrm{~h}$, the culture plates were taken out and added with $900 \mu \mathrm{L} \mathrm{RPMI} 1640$ cell culture medium containing $10 \%$ FBS, which was then put into the incubator for the following $72 \mathrm{~h}$ incubation.

\section{Morphologies of the cells cultured on the sponges}

The L929 cells cultured on the above EHSS-Pn and the negative control were rinsed with PBS solution twice and fixed with $2.5 \mathrm{wt} \%$ glutaraldehyde. Part of the fixed samples were stained with a $8 \mu \mathrm{g} / \mathrm{mL} \mathrm{DiO}$ solution, and then washed with PBS thrice. The sponges with DiO-stained cells were observed and photographed using a confocal microscope ( Leica-LCS-SP8-STED, Leica, Germany). The other part of the

fixed samples were dehydrated by immersing in $50,70,85,95$ and $100 \%(v / v)$ ethanol solution for 15 min in sequence, freeze-dried and coated with gold for SEM observation.

\subsection{Hemcompatibility evaluations of EHSS-Pn}


The hemocompatibility of is critical for their application as implants after implantation. Thus, the effects of EHSS-Pn on the blood coagulation time and components of blood should be evaluated systematically. The experimental processes were similar to our previous work (Zhao et al. 2018).

Firstly, the blood was taken from the heart of New Zealand rabbits (Wuhan University center for animal center experiment/A3 lab). The above blood was mixed with $3.8 \mathrm{wt} \%$ sodium citrate ( $1 / 9$ of the blood) to prepare anticoagulant whole blood, which was centrifuged for $15 \mathrm{~min}$. The upper plasma was then collected for the following use.

Secondly, the hemolysis test was performed. Briefly, a $2.0 \mathrm{~mL}$ fresh anticoagulant whole blood was diluted by adding into a $2.5 \mathrm{~mL}$ physiological saline. The $1 \times 1 \mathrm{~mm}$ EHSS-Pn sponges were put into the tubes and washed with distilled water for three times and rinsed with physiological saline, which was then replaced with $10 \mathrm{~mL}$ fresh physiological saline to keep at $37^{\circ} \mathrm{C}$ for $0.5 \mathrm{~h}$. The above tubes were kept for $1 \mathrm{~h}$ after the addition of $0.2 \mathrm{~mL}$ dilute whole blood, the resultant solutions in the tubes were then centrifuged. The absorbance of the corresponding supernatants was measured at $545 \mathrm{~nm}$. Distilled water or $0.9 \mathrm{wt} \%$ physiological saline $(10 \mathrm{~mL})$ were used to mix with $0.2 \mathrm{~mL}$ fresh whole blood and treated with the same process, which were coded as the positive and negative control, respectively. All the samples were repeated thrice, and the mean values were calculated. The hemolysis ratio (HR) was calculated by Eq. (2):

$\mathrm{HR}(\%)=(\mathrm{AS}-\mathrm{AN}) /(\mathrm{AP}-\mathrm{AN}) \times 100(2)$

Where AS, AN and AP are the average absorbance of EHSS-Pn, negative controls and positive controls, respectively.

Thirdly, the red blood cell incubation of the EHSS-Pn cells was conducted. The EHSS-Pn sponges were soaked in the physiological saline for $1 \mathrm{~h}$, which were taken out and squeezed to remove the physiological saline. $5 \mathrm{~mL}$ whole blood was diluted with $10 \mathrm{~mL}$ physiological saline, and then the desired amounts of diluted blood was added to the above saline-treated sponges, which were kept at $37^{\circ} \mathrm{C}$ for 1 h., The samples were fixed using $2.5 \%$ glutaraldehyde overnight after the removal of residual blood, washed with PBS for three times. The treated EHSS-Pn sponges were wrapped, freeze-dried and coated with gold for the following SEM observation with the accelerating voltage of $20 \mathrm{kV}$.

The last but not the least, the coagulation routine inspection including prothrombin time test (PT), activated partial thrombin time (APTT) and thrombin time (TT) was performed systematically. The EHSSPn sponges were cut into $2 \times 2 \times 2 \mathrm{~mm}$ blocks, placed in 96 well plates with the following addition of the above plasma ( $50 \mu \mathrm{L}$ per well). The incubation process were conducted by keeping the plates in a water bath pot for $10 \mathrm{~min}$. PT reagent (100 $\mu \mathrm{L}$ per well) was then added in the above plates and mixed immediately, and the plasma coagulation time was recorded as PT. Similarly, each wells with the EHSSPn sponges of same size was added with $50 \mu \mathrm{L}$ plasma and $50 \mu \mathrm{L}$ APTT reagent, and the blank control was set without sponges. After the incubation for $10 \mathrm{~min}, 50 \mu \mathrm{L}$ preheated $\mathrm{CaCl}_{2}$ reagent was added and blended immediately in each well, and the corresponding plasma coagulation time was recorded as 
APTT. Each wells with the same size EHSS-Pn sponges was added with $100 \mu \mathrm{L}$ plasma. After incubation for $10 \mathrm{~min}, 50 \mu \mathrm{L}$ preheated TT reagent was added in each well and mixed immediately, and the corresponding plasma coagulation time was recorded as TT. The wells were also treated by the above same three processes without the EHSS-Pn sponges, which were coded as the blank controls.

\section{Results And Discussion}

\subsection{Morphologies and structure of the EHSS-Pn Sponges}

The effect of the introduction of PPy on the morphologies for the surface of the HEC/SPI sponges was studied by SEM (Fig. 1). Obviously, all the EHSS-Pn ( $n=0,6$ and 12) sponges showed macro-porous structure with the pore size of about 150-250 $\mu \mathrm{m}$. The pore surface of EHSS-P0 were relative smooth with almost no particles (Fig. 1a), and many PPy nano-spheres appeared on the pore surface of EHSS-P6, indicating the successful synthesis of PPy on the HEC/SPI sponge matrix. The PPy nano-spheres assembled into abundant interconnected flocculent materials on the pore wall of EHSS-P12 with the further increase of the soaking time, which was anticipated to improve the electrical conductivity of the scaffold.

The morphologies and distribution of PPy on EHSS-Pn were further studied by TEM. There were some PPy nanospheres appeared on the for EHSS-P2, and the number and size of PPy nanospheres increased obviously in EHSS-P6, further indicating the successful synthesis of PPy on the sponges. The PPy nanospheres formed an interconnected structure for EHSS-P12 by the extension of the soaking time, which was consistent with the SEM result (Fig. 1f). Especially, the HEC/SPI matrix was almost covered with PPy completely to form a more interconnected structure (compared with EHSS-P12) in EHSS-P24, which was very important to improve their electrical conductivity.

The chemical composition and structure of EHSS-Pn were studied by FTIR and XRD. FTIR could be used to analyze the chemical structures of the sponge samples, and the FTIR spectra of the EHSS-Pn $(n=0$, 6,12 and 24) sponges are shown in Fig. 3a. The intensity of characteristic absorption bands of $\mathrm{C}=\mathrm{C}$ stretching for pyrrole ring and $\mathrm{C}-\mathrm{N}$ at around 1543 and $1040 \mathrm{~cm}^{-1}$ increased gradually from EHSS-PO to EHSS-P24, indicating the successful synthesis of PPy in the HEC/SPI sponge matrix (Huang et al. 2014; Manzari-Tavakoli et al. 2020; Bueno et al. 2015). The $-\mathrm{OH}$ absorption band at $3344 \mathrm{~cm}^{-1}$ for EHSS-PO shifted to the bands at around $3430 \mathrm{~cm}^{-1}$, indicating the strong hydrogen bonding interaction between HEC/SPI and PPy. Figure $3 b$ shows the XRD patterns of the EHSS-Pn $(n=0,6$ and 12$)$ sponges. The characteristic peaks for EHSS-P0 appeared at 8.2 and $19.7^{\circ}$, which was different from HEC and SPI, indicating the successful chemical crosslinking by ECH (Zhao et al. 2018). The peak intensity of these two peaks decreased slightly in EHSS-P6 and EHSS-P12 due to the amorphous state of the synthesized PPy (Shi et al. 2014).

\subsection{Mechanical properties and electrical conductivity of EHSS-Pn}


The effect of the incorporation of PPy on the mechanical properties of HEC/SPI sponges was studied by an universal material testing machine, and the results are shown in Fig. 4. Obviously, the compressive strength increased gradually from 0.16 MPa for EHSS-P0 to 0.43 MPa for EHSS-P24 with the increase of the soaking time, showing obvious stiffening effect due to the entanglement and intermolecular association between HEC/SPI matrix and PPy (Shi et al. 2014). Moreover, the strain of all the EHSS-Pn ( $\mathrm{n}$ $=0,4,12$ and 24) sponges remained almost unchanged at a high level of about $60 \%$ with the incorporation of PPy, this result indicated that the incorporated PPy didn't break the original HEC/SPI network, which was consisted with the result of SEM (Fig. 1).

Figure 5a shows the photograph of the EHSS-P12 sponge. The color of EHSS-P12 changed from white for HEC/SPI to black brown due to the incorporation of PPy. Obviously, PPy could distribute evenly on the HEC/SPI matrix to form a homogenous composite sponge, which could possibly endow the HEC/SPI sponge with electric conductivity. As we mentioned above, the improve of electrical conductivity for the $\mathrm{HEC} / \mathrm{SPI}$ sponges is critical to broaden their applications, which was evaluated by Four-Point Probes. Figure $5 b$ shows the conductivity values of EHSS-Pn $(n=0,1,2,4,6,12$ and 24$)$ sponges. Clearly, the electric conductivity increased sharply with the soaking time due to formation of interconnected PPy structure on the HEC/SPI matrix (Figs. 1 and 2). The conductivity value of EHSS-P1 was $0.0087 \mathrm{~S} / \mathrm{m}$, further indicating the successful introduction of PPy due to the non-conductivity of the HEC/SPI sponge (EHSS-P0). The conductivity values for EHSS-P2, EHSS-P4, EHSS-P6, EHSS-P12 and EHSS-P24 were $0.022,0.063,0.056,0.529$ and $0.885 \mathrm{~S} / \mathrm{m}$, respectively. It was noted that the conductivity of EHSS-P24 was more than two order of magnitude greater than that of EHSS-P1, showing high electrical conductivity (Shi et al. 2014). Thus, the incorporation of PPy improved the conductivity of the HEC/SPI sponge successfully.

\subsection{Cytocompatibility and hemcompatibility of EHSS-Pn}

In order to reveal the effect of the incorporation of PPy on the cytotoxicity of the HEC/SPI sponge, the cell viability of L929 for EHSS-Pn ( $n=0,1,2,4,6,12$ and 24) were evaluated by MTT assay. Figure 6a shows the results of the cytotoxicity tests for EHSS-Pn $(n=0,1,2,4,6,12$ and 24). Obviously, almost all the cell viability values of EHSS-Pn sponges (except EHSS-P1) had no statistical difference (*P囚0.05) compared with the control, showing no cytotoxicity with the incorporation of PPy. Interestingly, the cell viability values for EHSS-P1 at $24 \mathrm{~h}$ and $72 \mathrm{~h}$ were a little higher than those of the control and had statistical difference ( $\left.{ }^{\star} P<0.05\right)$, showing no cytotoxicity and the improvement effect on cell proliferation. The biocompatibility of EHSS-Pn sponges were evaluated by direct contact method. The 3D images of L929 cells cultured on for $72 \mathrm{~h}$ are shown in Fig. $6 \mathrm{~b}$. Obviously, cells could adhere and proliferate on both the surface and interiors of all the EHSS-Pn ( $n=0,4,12$ and 24) sponges due to the macro-porous structure (Fig. 1), which were further confirmed in Movie S1 (Supporting Information) for EHSS-P12, indicating good biocompatibility. Moreover, cell shape changed gradually from round for EHSS-P4 to shuttle shape for EHSS-P24, indicating that the incorporation of PPy was better for cell adhesion and proliferation.

To further evaluate the biocompatibility of EHSS-Pn, the adhesion, proliferation and distribution of L929 cells on the EHSS-Pn ( $n=0,1,2,4,6,12$ and 24) sponges after $72 \mathrm{~h}$ direct contact were also observed by 
SEM, and the corresponding images are shown in Fig. 7. Abundant L929 cells distributed homogenously both on the surface and internal of all the EHSS-Pn sponges due to their macro-porous structure. indicating good cell adhesive ability. The proportions of shuttle-like L929 cells on the EHSS-Pn sponges increased gradually from EHSS-P0 to EHSS-P12 on the whole. Moreover, most of the L929 cells exhibited shuttle-like shape on the sponges, further confirming their good biocompatibility.

Table 1

Erythrocyte Hemolysis of EHSS-Pn ( $n=0,1,2,4,6$, 12 and 24) sponges

\begin{tabular}{|ll|}
\hline & HR(\%) \\
\hline Distilled water (Positive control) & 100 \\
\hline Normal saline (Negative control) & 0 \\
\hline EHSS-P0 & $0.96 \pm 0.55$ \\
\hline EHSS-P1 & $1.08 \pm 0.62$ \\
\hline EHSS-P2 & $0.92 \pm 0.41$ \\
\hline EHSS-P4 & $1.13 \pm 0.46$ \\
\hline EHSS-P6 & $1.5 \pm 0.42$ \\
\hline EHSS-P12 & $1.64 \pm 0.53$ \\
\hline EHSS-P24 & $2.74 \pm 0.43$ \\
\hline
\end{tabular}

Generally, the basic requirement of materials for use to contact with blood is that their hemolysis rate should be below $5 \%$. Table 1 shows the hemolysis rates of the groups of negative control, positive control and the EHSS-Pn sponges, respectively. Lower hemolysis rate for biomedical materials means less destruction to red blood cells. The hemolysis rates of all the EHSS-Pn $(n=0,1,2,4,6,12$ and 24) sponges were below $5 \%$, showing no hemolysis and good hemcompatibility. So all the EHSS-Pn sponges could be used as biomaterials to contact with blood cells directly.

The red blood cell incubation tests were conducted to further evaluate the hemocompatibility of EHSS-Pn. Figure 8 shows the SEM morphologies of the EHSS-Pn $(n=0,2,4,6,12$ and 24) sponges co-cultured with the New Zealand rabbit whole blood. Obviously, the vast majority of the red blood cells on all the EHSSPn sponges remained biconcave shape after co-culture with the EHSS-Pn sponges, showing no obvious difference with the normal red blood cells in the anticoagulant blood. So the introduction of PPy into the $\mathrm{HEC} / \mathrm{SPI}$ matrix would not destroy the red blood cells and have obvious negative effect on the red blood cells, which is the largest number of cells in the blood, indicating good hemocompatibility.

Moreover, coagulation routine inspection was further conducted. The exogenous coagulation system status could be reflected by $\mathrm{PT}$, the congenital or acquired fibrin prothrombin and the loss of $\nabla, \nabla$ and $\nabla$ 
blood coagulation factors or the presence of inhibitor could also be verified by PT. The result of routine blood coagulation of EHSS-Pn ( $n=0,1,2,4,6,12$ and 24) through PT method is shown in Fig. 9a. The gap of PT values between all the EHSS-Pn sponges and the blank control were all less than $3 \mathrm{~s}$, indicating that components of the EHSS-Pn sponges had almost no effect on the exogenous coagulation. The endogenous blood coagulation system status could be reflected by APTT, the in vivo concentrations of

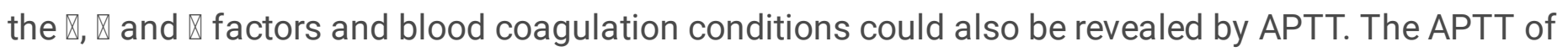
EHSS-Pn ( $n=0,1,2,4$ and 6) showed no significant difference compared with the blank control group (Fig. 9b), indicating no obvious influence on the of blood. However, the APTT value of EHSS-P12 and EHSS-P24 were smaller than that of the blank control, which was possibly due to the change of the concentration of some components during the endogenous clotting with the increase of the PPy content. The conversion time from fibrinogen to fibrin could be reflected by TT. As seen from Fig. 9c, the TT of EHSS-Pn ( $n=0,1,2,4,6,12$ and 24) was close to or smaller than the blank control group, so there was no clinical significance according to the clinical significance of TT (Zhao et al. 2018). Therefore, the EHSS-Pn ( $n=0,1,2,4,6,12$ and 24) sponges had good biocompatibility and hemocompatibility, showing potential application as implants with electrical conductivity.

\section{Conclusions}

A series of electro-conductive EHSS-Pn ( $n=0,1,2,4,6,12$ and 24) were successfully fabricated by in-situ synthesis PPy on the HEC/SPI sponge matrix. The results indicated that EHSS-Pn still exhibit homogenous inter-connected macroporous structure with pore size of about 150-250 $\mu \mathrm{m}$, indicating that the incorporation of PPy didn't break the original sponge structure. The evenly distributed PPy significantly increased the mechanical properties and electrical conductivity of the HEC/SPI sponge, the compressive strength and conductivity values for EHSS-P24 could reach as high as $0.43 \mathrm{MPa}$ and 0.885 $\mathrm{S} / \mathrm{m}$, respectively. Moreover, EHSS-Pn had good cytocompatibility and hemocompatibility with no significant side effects on the anticoagulant whole blood. Therefore, the composite electro-conductive sponges showed potential application in the tissue engineering field that require electrical conductivity for stimulation or sensing such as neural tissue restoration.

\section{Declarations}

Acknowledgments. This work was supported by the National Natural Science Foundation of China(Grant Nos: NSFC 81871493 and 51903217), Medical Science and Technology Research Plan Joint Construction Project of Henan Province (2018020121), the Medical Science Advancement Program (Clinical Medicine) of Wuhan University (TFLC2018003), and the Natural Science Foundation of the Jiangsu Higher Education Institutions of China $\triangle$ Grant No. 20KJB430032区. Thanks for the technique support from the Experimental Teaching Center of Basic Medical Sciences, Wuhan University.

\section{Compliance with ethical standards}


Conflict of interest: The authors declare that they have no conflict of interest.

Ethical approval: The study was approved by the Ethics Committees of The First Affiliated Hospital of Zhengzhou University, Wuhan University and Yancheng Institute of Technology. All animal experimental procedures were carried out in accordance with the Guidelines and Regulations for Care and Use of Laboratory Animals based on the Experimental Animal Management Ordinance (National Science and Technology Committee of the People's Republic of China, 1998) and with the Guideline for Care and Use of Laboratory Animal of Wuhan University. The experiments were approved by the Animal Ethics Committee of School of Basic Medical Sciences of Wuhan University and carried out at the Wuhan University Laboratory Animal Center. The article does not contain any experiments with human participants performed by all of the authors.

Informed consent: Informed consent was obtained from all individual participants included in the study.

\section{References}

Abbasian M, Massoumi B, Mohammad-Rezaei R, Samadian H, Jaymand M (2019) Scaffolding polymeric biomaterials: Are naturally occurring biological macromolecules more appropriate for tissue engineering? Int J Biol Macromol 134:673-694. https://doi.org/10.1016/j.ijbiomac.2019.04.197

Ahadian S, Huyer LD, Estili M, Yee B, Nathaniel S, Xu Z, Sun Y, Radisic M (2017) Moldable elastomeric polyester-carbon nanotube scaffolds for cardiac tissue engineering. Acta Biomater 52: 81-91. https://doi.org/10.1016/j.actbio.2016.12.009

Alegret N, Dominguez-Alfaro A, Mecerreyes D (2019) 3D scaffolds based on conductive polymers for biomedical applications. Biomacromolecules 20:73-78. https://doi.org/10.1021/acs.biomac.8b01382

Bahrami S, Solouk A, Mirzadeh H, Seifalian AM (2019) Electroconductive polyurethane/graphene nanocomposite for biomedical applications. Compos Part B-Eng 168:421-431.

https://doi.org/10.1016/j.compositesb.2019.03.044

Baniasadi H, Ramazani A. S. A, Mashayekhan S (2015) Fabrication and characterization of conductive chitosan/gelatin-based scaffolds for nerve tissue engineering. Int J Biol Macromol 74: 360-366. https://doi.org/10.1016/j.ijbiomac.2014.12.014

Bueno VB, Takahashi SH, Catalani LH, DeTorresi SIC, Petri DFS (2015). Biocompatible xanthan/polypyrrole scaffolds for tissue engineering. Mat Sci Eng C-Mater 52:121-128. https://doi.org/10.1016/j.msec.2015.03.023

Clayton RH, Bernus O, Cherry EM, Dierckx H, Fenton FH, Mirabella L, Panfilov AV, Sachse FB, Seemann G, Zhang H (2011) Models of cardiac tissue electrophysiology: Progress, challenges and open questions. Prog Biophys Mol Bio 104 (1-3): 22-48. https://doi.org/10.1016/j.pbiomolbio.2010.05.008 
Deng Z, Hu T, Lei Q, He J, Ma PX, Guo B (2019) Stimuli-Responsive Conductive Nanocomposite Hydrogels with High Stretchability, Self-Healing, Adhesiveness, and 3D Printability for Human Motion Sensing. ACS Appl Mater Interfaces 11: 6796-6808. http://dx.doi.org/10.1021/acsami.8b20178

Dong R, Ma PX, Guo B (2019) Conductive Biomaterials for Muscle Tissue Engineering. Biomaterials 229:119584. https://doi.org/10.1016/j.biomaterials.2019.119584

Du Y, Ge J, Li Y, Ma PX, Lei B (2018) Biomimetic elastomeric, conductive and biodegradable polycitratebased nanocomposites for guiding myogenic differentiation and skeletal muscle regeneration. Biomaterials 157:40-50. https://doi.org/10.1016/j.biomaterials.2017.12.005

Funk RHW, Monsees T, Zkucur N (2009) Electromagnetic effects - From cell biology to medicine. Prog Histochem Cyto 43(4): 177-264. https://doi.org/10.1016/j.proghi.2008.07.001

He M, Wang Z, Cao Y, Zhao Y, Duan B, Chen Y, Xu M, Zhang L (2014) Construction of chitin/PVA composite hydrogels with jellyfish gel-like structure and their biocompatibility. Biomacromolecules 15(9): 3358-3365. http://dx.doi.org/10.1021/bm500827q

Huang ZB, Yin GF, Liao XM, Gu JW (2014) Conducting polypyrrole in tissue engineering applications. Front Mater Sci $8: 39-45$. http://dx.doi.org/10.1007/s11706-014-0238-8

Hung TJ, Ostrovsky-Snider NA, Patrick YDM, Donohue JD, Seob CJ, Rakchanok C, Larson JD, Ruth MA, Deok-Ho K (2018) Conductive Silk-Polypyrrole Composite Scaffolds with Bioinspired Nanotopographic Cues for Cardiac Tissue Engineering, Mater Chem B 6: 7185-7196.

http://dx.doi.org/10.1039/C8TB01116H

Kashi M, Baghbani F, Moztarzadeh F, Mobasheri H, Kowsari E (2018) Green synthesis of degradable conductive thermosensitive oligopyrrole/chitosan hydrogel intended for cartilage tissue engineering. Int J Biol Macromol 107:1567-1575. https://doi.org/10.1016/j.ijbiomac.2017.10.015

Kayser LV, Lipomi DJ (2019) Stretchable Conductive Polymers and Composites Based on PEDOT and PEDOT:PSS, Adv Mater 31:1806133.

Khorshidi S, Karkhaneh A (2018) Hydrogel/fiber conductive scaffold for bone tissue engineering. J Biomed Mater Res A 3:718-724. https://doi.org/10.1002/jbm.a.36282

Kim S, Jang LK, Jang M, Lee S, Hardy JG, Lee JY (2018) Electrically Conductive PolydopaminePolypyrrole as High Performance Biomaterials for Cell Stimulation in Vitro and Electrical Signal Recording in Vivo. ACS Applied Materials \& Interfaces 10: 33032-33042. https://doi.org/10.1021/acsami.8b11546

Manzari-Tavakoli A, Tarasi R, Sedghi R, Moghimi A, Niknejad H (2020) Fabrication of nanochitosan incorporated polypyrrole/alginate conducting scaffold for neural tissue engineering. Sci Rep 10:22012. http://dx.doi.org/10.1038/s41598-020-78650-2 
Massoumi B, Abbasian M, Jahanban-Esfahlan R, Mohammad-Rezaei R, Khalilzadeh B, Samadian H, Rezaei A, Derakhshankhah H, Jaymand M (2020) A novel bio-inspired conductive, biocompatible, and adhesive terpolymer based on polyaniline, polydopamine, and polylactide as scaffolding biomaterial for tissue engineering application. Int J Biol Macromol 147: 1174-1184.

https://doi.org/10.1016/j.ijbiomac.2019.10.086

Milakin KA, Acharya U, Bober P (2020) Biocompatible and antibacterial gelatin-based polypyrrole cryogels. Polymer 197:122491. https://doi.org/10.1016/j.polymer.2020.122491

Min J, Patel M, Koh WG (2018) Incorporation of Conductive Materials into Hydrogels for Tissue Engineering Applications. Polymers 10(10): 1078. https://doi.org/10.3390/polym10101078

Qu J, Zhao X, Liang Y, Xu Y, Ma P X, Guo B (2019) Degradable conductive injectable hydrogels as novel antibacterial, antioxidant wound dressings for wound healing. Chem Eng J 362:548-560.

https://doi.org/10.1016/j.cej.2019.01.028

Sadeghi A, Moztarzadeh F, Mohandesi JA (2018) Investigating the effect of chitosan on hydrophilicity and bioactivity of conductive electrospun composite scaffold for neural tissue engineering. Int $\mathrm{J}$ Biol Macromol 121: 625-632. https://doi.org/10.1016/j.ijbiomac.2018.10.022

Shi Z, Gao H, Feng J, Ding B, Cao X, Kuga S, Cai J (2014) In Situ Synthesis of Robust Conductive Cellulose/Polypyrrole Composite Aerogels and Their Potential Application in Nerve Regeneration. Angew Chem Int Edit 126 (21):5484-5488. http://dx.doi.org/10.1002/ange.201402751

Su D, Zhou J, Ahmed KS, Ma Q, Lv G, Chen J (2019) Fabrication and characterization of collagen-heparinpolypyrrole composite conductive film for neural scaffold. Int J Biol Macromol 129:895-903. https://doi.org/10.1016/j.jjbiomac.2019.02.087

Walker BW, Lara RP, Mogadam E, Yu CH, Kimball W, Annabi N (2019) Rational Design of Microfabricated Electroconductive Hydrogels for Biomedical Applications. Prog Polym Sci 92: 135-157. https://doi.org/10.1016/j.progpolymsci.2019.02.007

Wang S, Sun C, Guan S, Li W, Xu J, Ge D, Zhuang M, Liu T, Ma X (2017) Chitosan/gelatin porous scaffolds assembled with conductive poly(3,4-ethylenedioxythiophene) nanoparticles for neural tissue engineering. J Mater Chem B 5:4774-4788. http://dx.doi.org/10.1039/C7TB00608J

Zamora-Sequeira R, Ardao I, Starbird R, García-González CA (2018) Conductive nanostructured materials based on poly-(3,4-ethylenedioxythiophene) (PEDOT) and starch/k-carrageenan for biomedical applications. Carbohyd Polym 189:304-312. https://doi.org/10.1016/j.carbpol.2018.02.040

Zhao Y, He M, Jin H, Zhao L, Du Q, Deng H, Tian W, Li Y, Lv X, Chen Y (2018) Construction of highly biocompatible hydroxyethyl cellulose/soy protein isolate composite sponges for tissue engineering. Chem Eng J 341: 402-413. https://doi.org/10.1016/j.cej.2018.02.046 
Zhao Y, Li Y, Du Q, Zhang Q, Lv X, Yang Q, Chang PR, Anderson DP, He M, Chen Y (2019) Shape memory histocompatible and biodegradable sponges for subcutaneous defect filling and repair: greatly reducing surgical incision. J Mater Chem B 7 (38): 5848-5860. http://dx.doi.org/10.1039/C9TB00902G

\section{Figures}
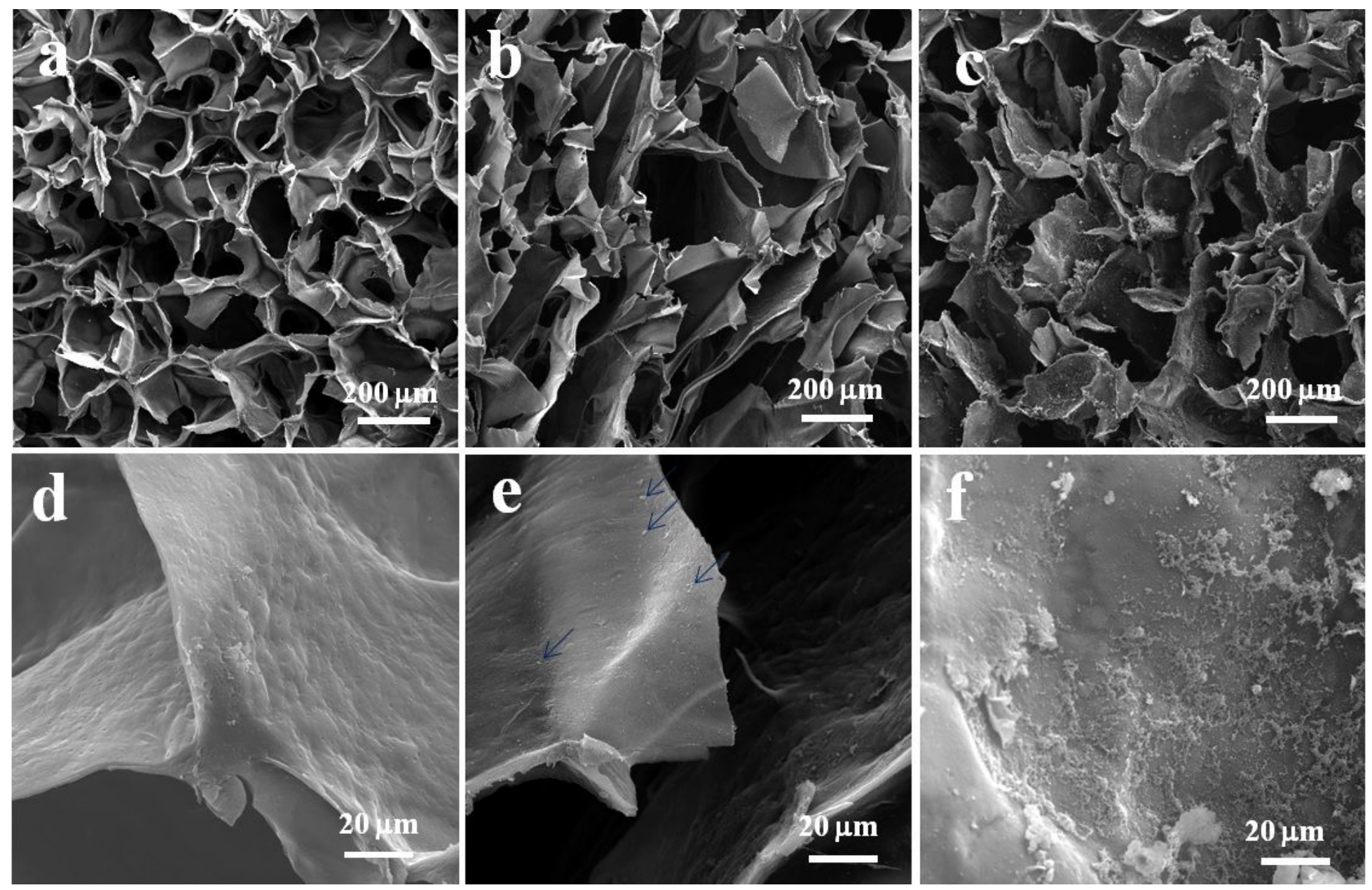

\section{Figure 1}

SEM images of the surfaces of EHSS-P0 (a, d), EHSS-P6 (b, e) and EHSS-P12 (c, f) sponges. The blue arrows indicate PPy nano-spheres. 


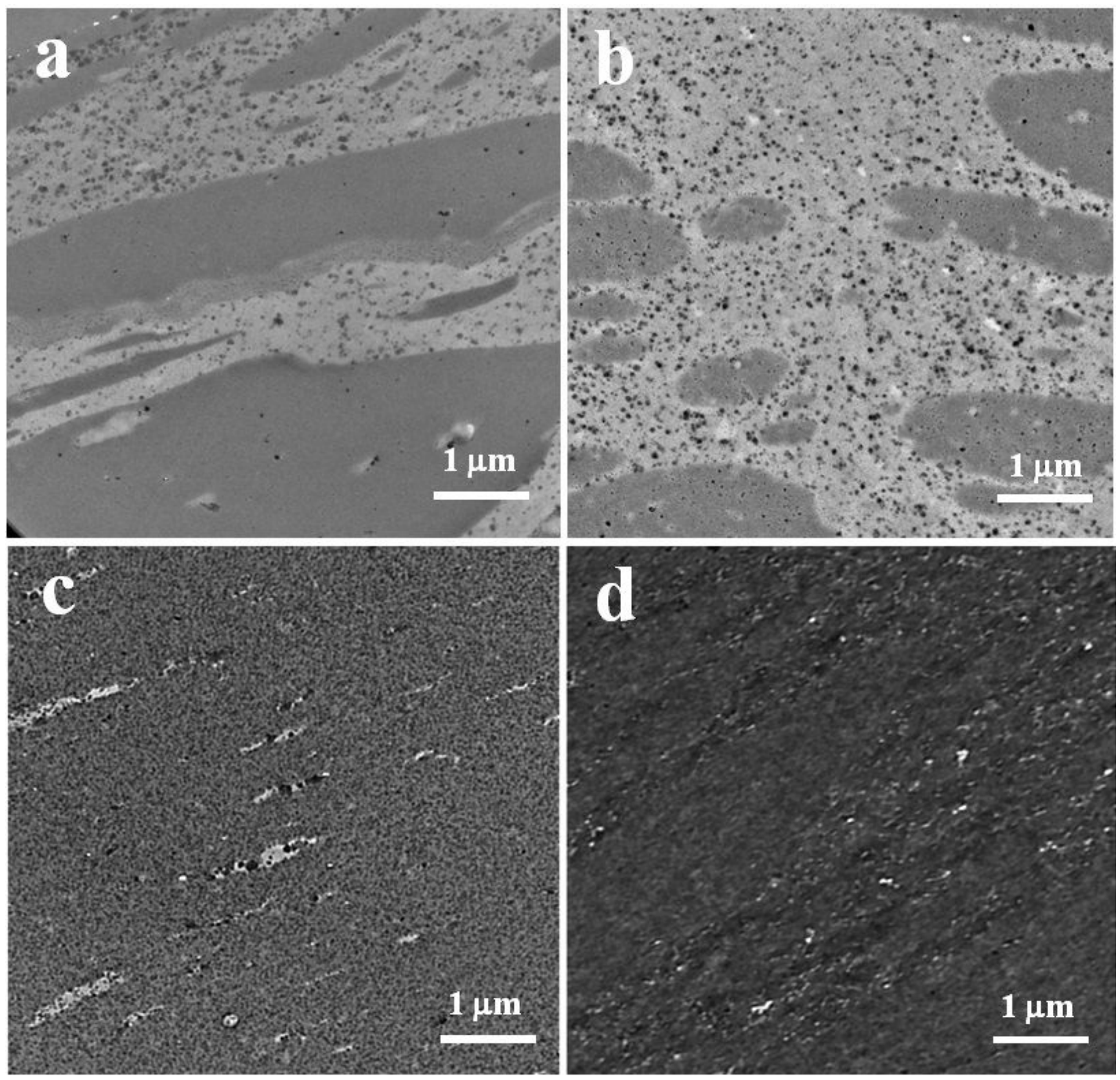

Figure 2

TEM images of the ultrathin sections of the EHSS-P2 (a), EHSS-P6 (b), EHSS-P12 (c) and EHSS-P24 (d) sponges. 

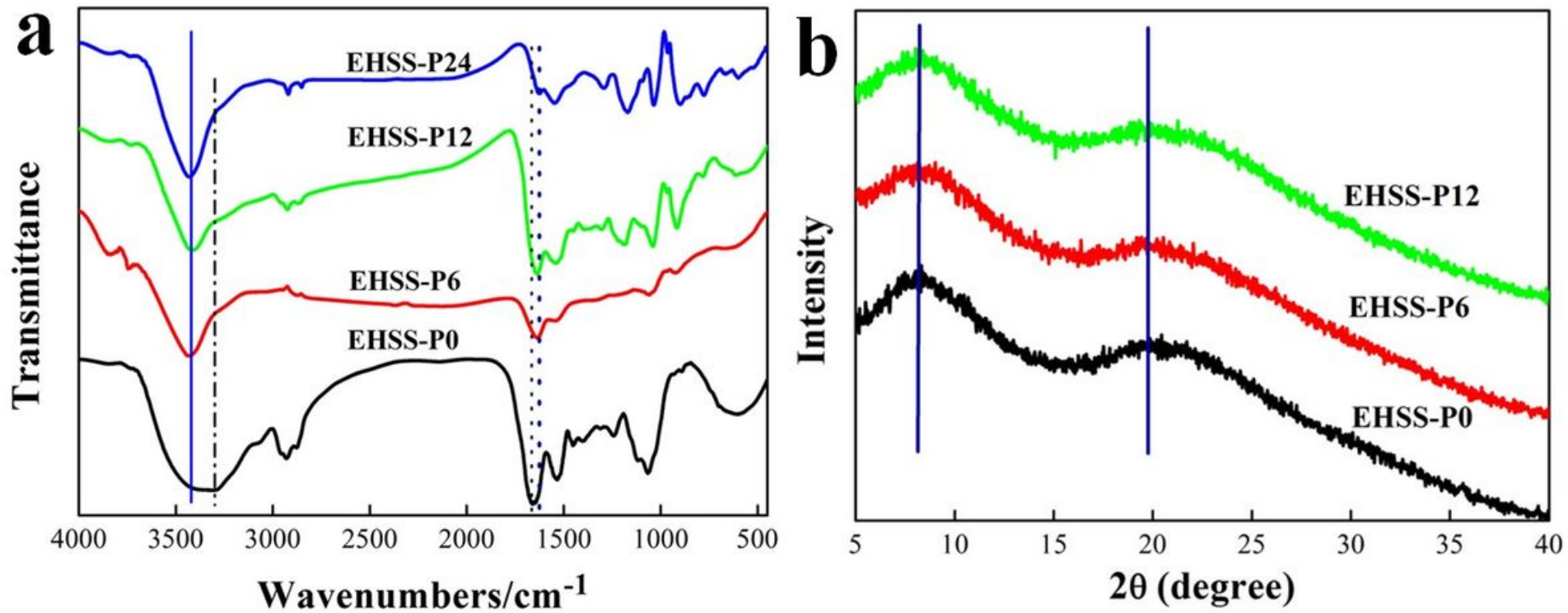

Figure 3

FTIR spectra of the EHSS-Pn $(n=0,6,12$ and 24) sponges (a), and XRD patterns of EHSS-Pn $(n=0,6$ and 12) sponges (b).
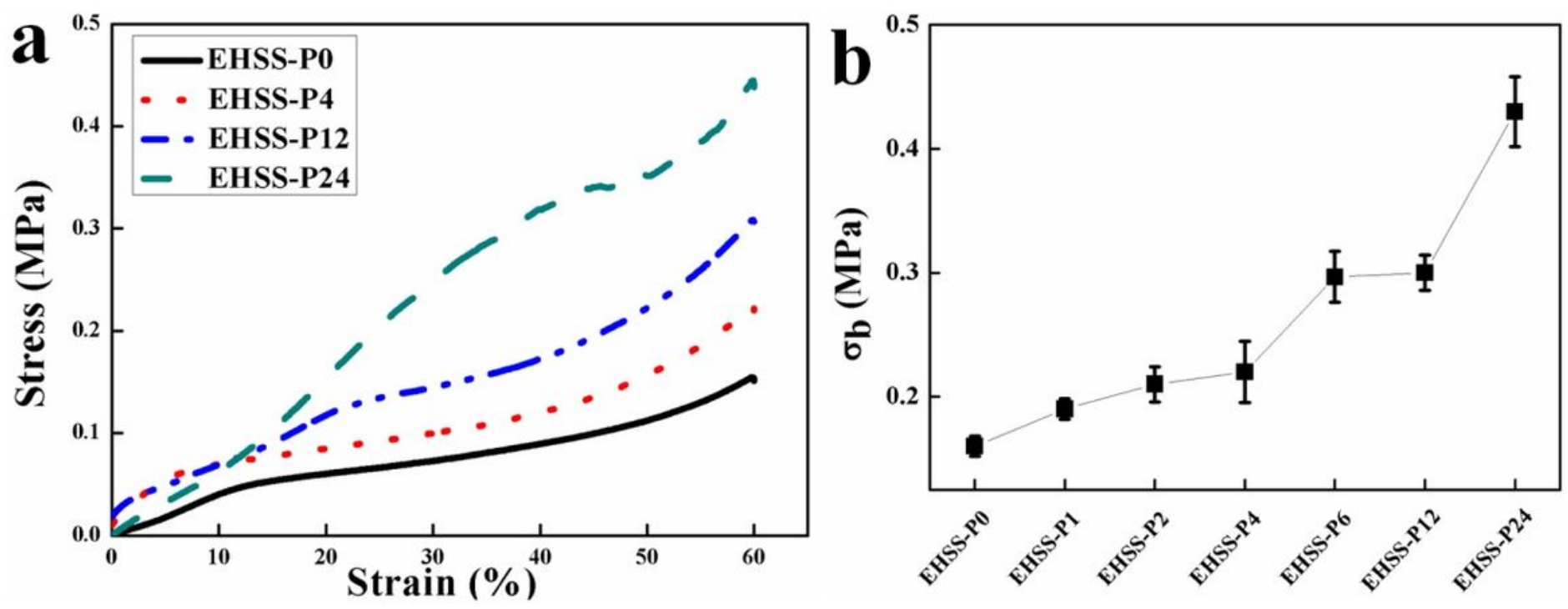

Figure 4

The compressive stress-strain curves of EHSS-Pn ( $n=0,4,12$ and 24) sponges (a) and corresponding compressive strength ( $\sigma b)(b)$ of EHSS-Pn $(n=0,1,2,4,6,12$ and 24) sponges. 

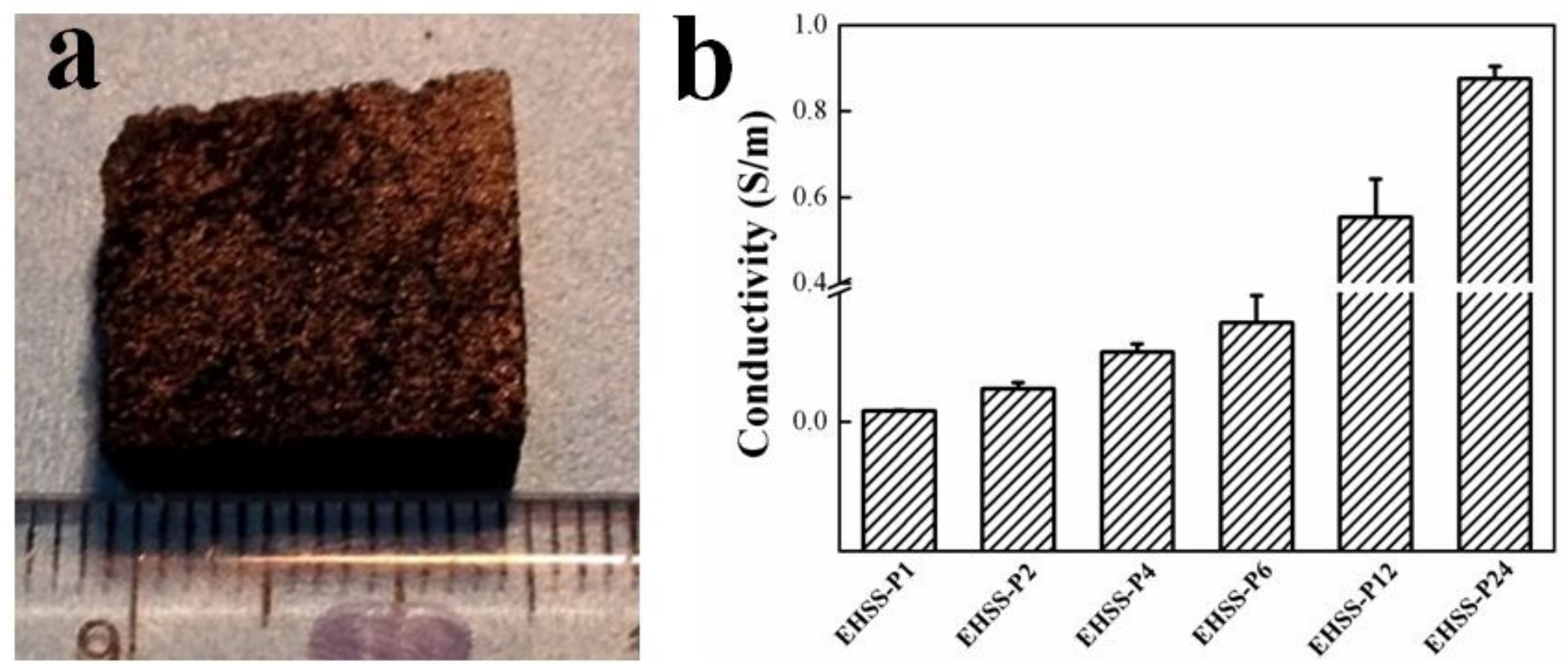

Figure 5

The photograph of EHSS-P12 (a) and the conductivity of EHSS-Pn ( $n=0,1,2,4,6,12$ and 24) sponges (b).
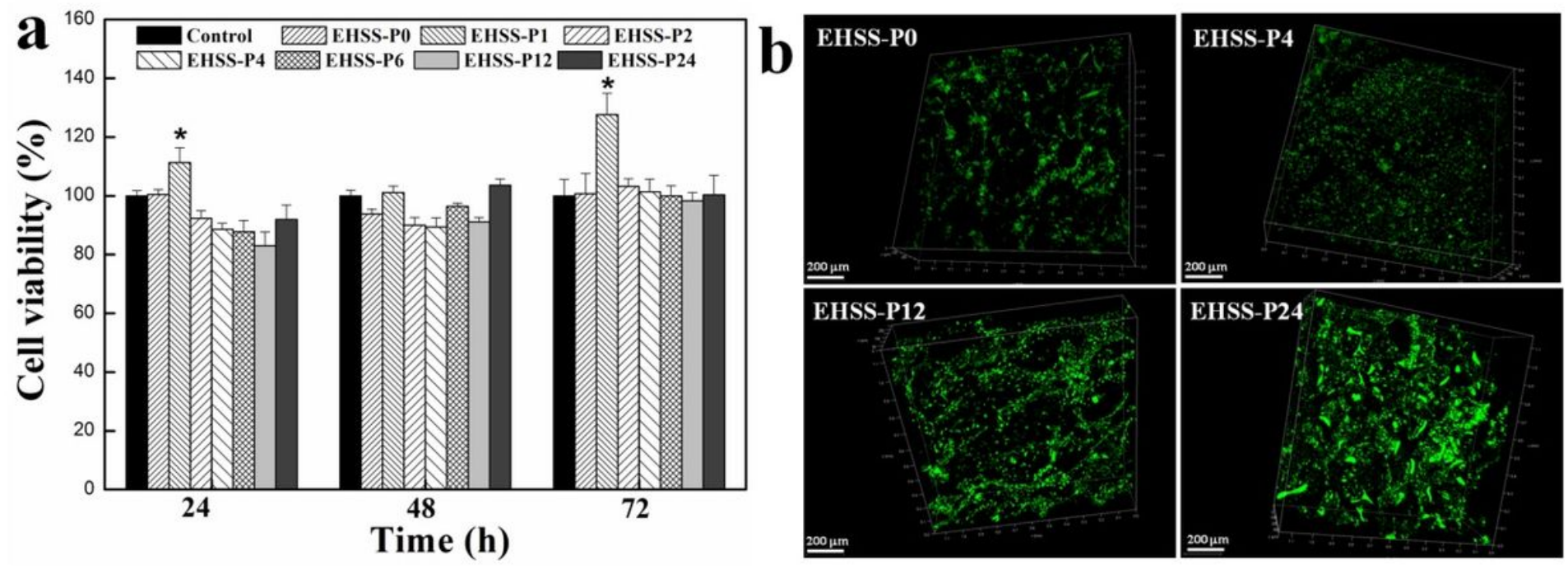

Figure 6

Cell viability of L929 cultured in extracts from EHSS-Pn $(n=0,1,2,4,6,12$ and 24$)$ for 24,48 and 72 h. *P $<0.05$ (compared with the control at the same time) (a), and confocal microscope 3D images of L929 cells (stained with DiO) cultured on the EHSS-Pn ( $n=0,4,12$ and 24) sponges for $72 \mathrm{~h}(\mathrm{~b})$. 


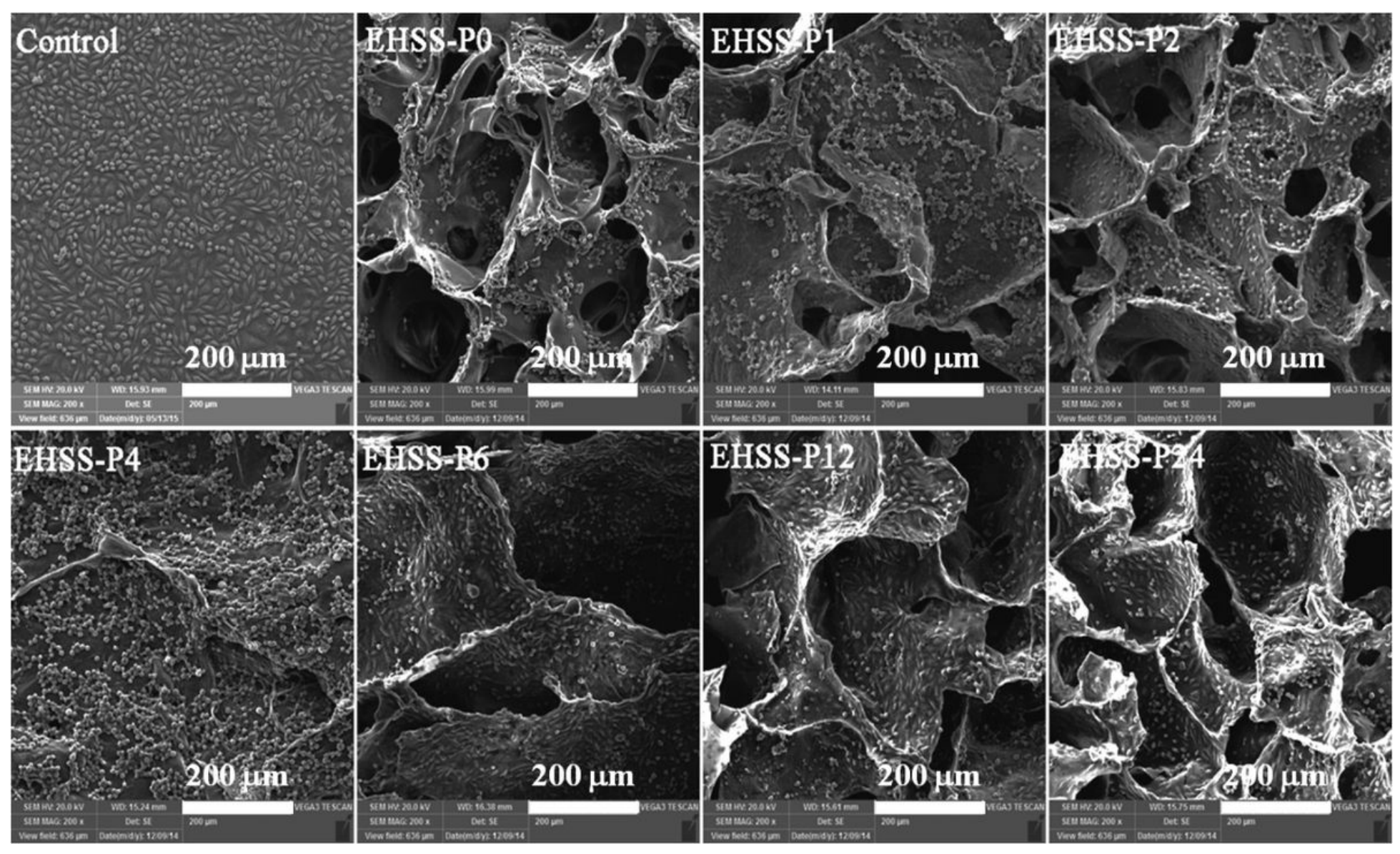

Figure 7

SEM images of L929 cells cultured on the surfaces of the EHSS-Pn $(n=0,1,2,4,6,12$ and 24) sponges for $72 \mathrm{~h}$. 

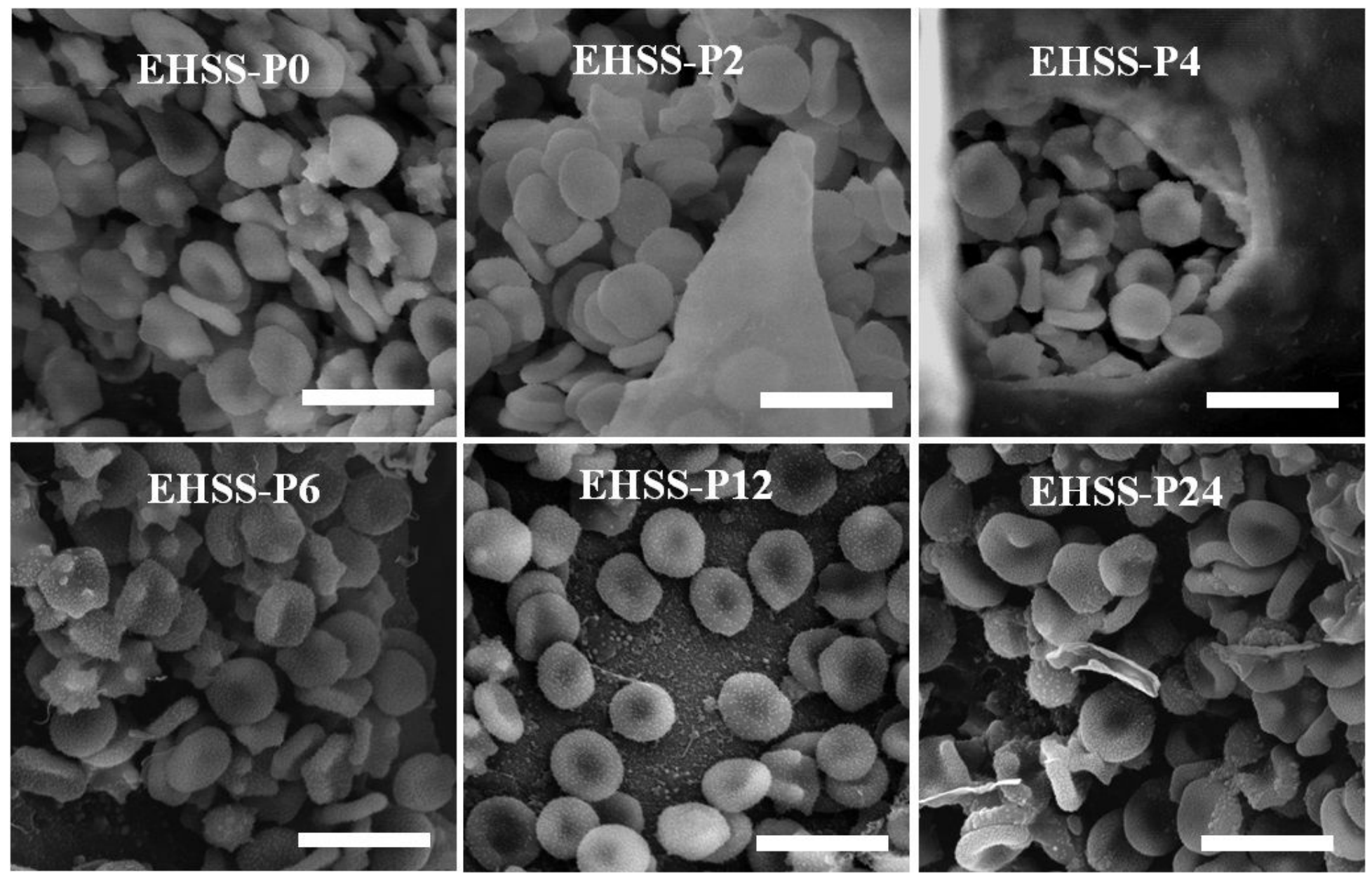

Figure 8

The SEM morphologies of red blood cell contact with the EHSS-Pn $(n=0,2,4,6,12$ and 24) sponges for $1 \mathrm{~h}$. Scale bars $=10 \mathrm{um}$.
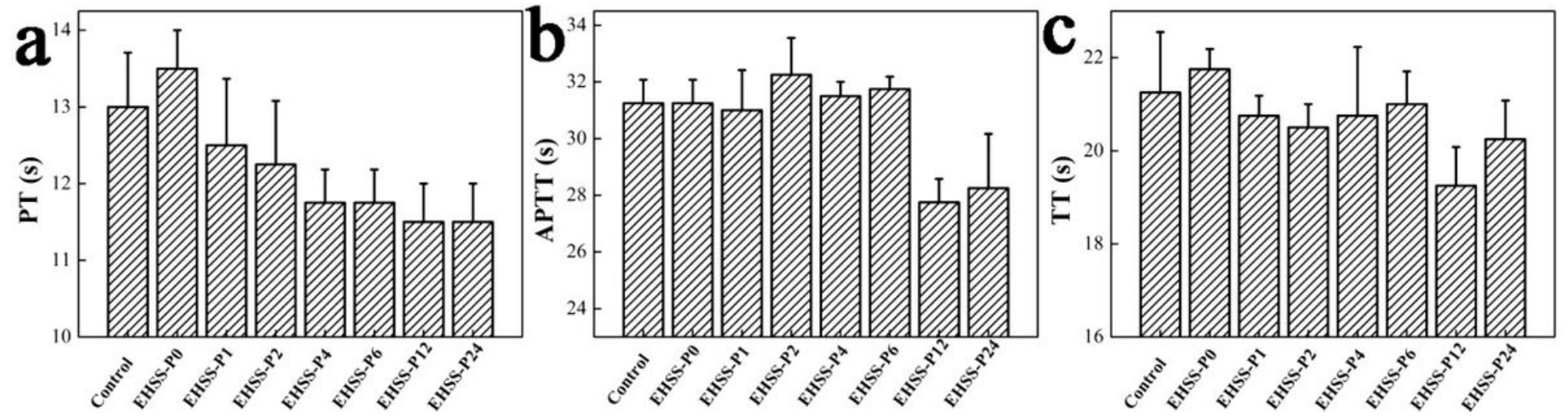

Figure 9

Coagulation routine of EHSS-Pn ( $n=0,1,2,4,6,12$ and 24$)$ sponges. PT(a), APTT(b) and TT(c). *P<0.05 (EHSS-Pn compared to control).

\section{Supplementary Files}


This is a list of supplementary files associated with this preprint. Click to download.

- MovieS1SupporptingInformation.mp4 